Article

\title{
Foreign vector measures
}

\author{
Abalo Douhadji ${ }^{1, *}$ and Yaovi Awussi ${ }^{2}$ \\ 1 Department of Mathematics, University of Lomé, PObox 1515, Lomé, Togo. \\ 2 Department of Mathematics, Mathematics and Applications Laboratory, University of Lomé, PObox 1515, Lomé, \\ Togo. \\ * Correspondence: douhadjiabalo@gmail.com
}

Received: 1 March 2020; Accepted: 20 April 2020; Published: 16 August 2020.

\begin{abstract}
We study the foreign measures in general by proving all operations possibilities with their characteristic relation $\perp$ and deduce that the set of foreign vector measures is a subset of bounded vector measures; stable par linear combination.
\end{abstract}

Keywords: Vector measure, Haar measure, foreign measure, absolute continuity.

MSC: 28C10, 28A05, 22C05, 03E17.

\section{Introduction}

T $n$ 2019, Monfort [1] wrote an article on the importance of foreign positive measure. Radon-Nikodym-Lebesgue showed that any positive, $\sigma$-finite measure can be decomposed uniquely to two measures; absolutely continue measure and the foreign measure [2-4]. Recently, in [5], Douhadji and Awussi showed that if we take $K(G, E)$, the space of continuous function with compact support on $G$, any vector measure $m$ on $K(G, E)$ decomposes uniquely into:

$$
m=m_{a}+m_{s} \quad \text { with } \quad m_{a} \ll \mu \text { and } m_{s} \perp \mu
$$

where $m_{a} \ll \mu$ means that $m_{a}$ is absolutely continue with respect to Haar measure $\mu[4,6]$.

In this paper, we prove that the set of foreign vector measures endowed with addition and their characteristic relation $\perp$ is stable par linear combination. This paper is organized as follows: in Section 2, we give some definitions related to vector measure and foreign measures, then we give some essentials theorems which help us to arrive to the expected theorem. In the Section 3 we present our main result.

\section{Preliminaries}

In this section, we give basic definitions of concepts concerning a vector measure, foreign measure and some important results. We fixed the following notions:

- $E$ and $F$ are Banach spaces;

- $G$ a locally compact group;

- $K(G, E)$ the space of continuous function with compact support on $G$;

- $M(G)$ the vector space of bounded vector measure;

- $\mathfrak{M}(G)$ the subset of $M(G)$ composed of foreign vector measures.

Our work in this article is to show the possible operations in the set $\mathfrak{M}(G)$.

Definition 1. Let $G$ be a locally compact group and $K(G ; E)$ be the space of $E$ valued functions with compact support on $G$. A vector measure on $G$ with respect to Banach spaces $E$ and $F$ is a linear map:

$$
\begin{aligned}
m: \quad K(G ; E) & \rightarrow F \\
f & \mapsto m(f)
\end{aligned}
$$

such as $\forall K$ compact in $G \exists a_{K}>0,\|m(f)\|_{F} \leq a_{K}\|f\|_{\infty}$, where \|\|$_{F}$ is the norm on Banach spaces $F$ and $\|f\|_{\infty}=\sup \left\{\|f(t)\|_{E}, t \in G\right\}$ is the norm on $K(G ; E)$. 
Definition 2. [2] A map $m$ is called a finitely additive vector measure or simply vector measure if whenever $A$ and $B$ are disjoint subset of $G$ then:

$$
m(A \cup B)=m(A)+m(B) .
$$

If $\left(A_{i}\right)_{1 \leq i \leq n}$ are pairwise disjoint subsets of $G$, we have:

$$
m\left(\bigsqcup_{i=1}^{n=\infty} A_{i}\right)=\sum_{i=1}^{\infty} m\left(A_{i}\right) .
$$

We note that [2]:

$$
m(A)=\int 1_{A} d m
$$

Let $m$ be a vector measure. The variation of $m$ is the nonnegative function $\|m\|$ whose value on a set $A \subset G$ is given by:

$$
\|m\|(A)=\sup \sum_{B \in \pi}\|m(B)\|,
$$

where the supremum is taken over all partitions $\pi$ of $A$.

We know that

$$
\|m\|(A)=0 \Leftrightarrow m(A)=0 .
$$

Let $\mu$ be positive and $\sigma$ finite and $v$ complex and $\sigma$ finite. Now, we give the definition of foreign positive measure.

Definition 3. [1,3] Two measures $v$ and $\mu$ are foreign (or singular or separate) if there exists a partition $\left(E_{1}, E_{2}\right)$ of $E$ such as $|\mu|\left(E_{1}\right)=0$ and $|v|\left(E_{2}\right)=0$. Note that $\mu \perp v$.

Proposition 1. [1,3] Two measures $v$ and $\mu$ are foreign if there exists measurable set $A$ such as

$$
|\mu|(A)=0 \text { and }|v|(\bar{A})=0 .
$$

Proposition 2. [1,3] Two measures $v$ and $\mu$ are foreign if there exists measurable set $A$ such as $A$ carries $\mu$ and $\bar{A}$ carries $v$.

The following theorem proved in [5] allows us to use the previous properties for vector measures.

Theorem 1. Let $E$ and $F$ are two Banach spaces and $G$ a locally compact group. Let $v \in K(G, E)$ be a complex measure which is $\sigma$-finite and $w \in F$ a nonzero vector. The mapping

$$
\begin{aligned}
m: \quad K(G, E) & \rightarrow F \\
f & \mapsto w v(f) \text { is a vector measure. }
\end{aligned}
$$

Now, we are able to give the following theorem:

Theorem 2. Let $m_{1}$ and $m_{2}$ belong to $\mathfrak{M}(G)$, then

$$
m_{1} \perp m_{2} \Longleftrightarrow \exists A \subset G\left\|m_{1}\right\|(A)=0 \text { and }\left\|m_{2}\right\|(\bar{A})=0 .
$$

Proof. Suppose $m_{1} \perp m_{2}$, then from Theorem 1, we have $m_{1}(f)=w v_{1}(f)$ and $m_{2}(f)=w v_{2}(f) ; \forall f \in$ $K(G ; E) ; w \neq 0$. So $v_{1} \perp v_{2}$. Now by Proposition 1 , there exists $A \subset G$ such as

$$
v_{1}(A)=0 \text { and } v_{2}(\bar{A})=0,
$$

which is equivalent to

$$
w v_{1}(A)=0 \text { and } w v_{2}(\bar{A})=0,
$$

which is equivalent to

$$
m_{1}(A)=0 \text { and } m_{2}(\bar{A})=0 .
$$


Now from (2), we have $\left\|m_{1}\right\|(A)=\sup \left\{\left\|m_{1}(B)\right\| ; B \subset A\right\}$, so $\left\|m_{1}\right\|(A)=0$. Similarly we can have $\left\|m_{2}\right\|(\bar{A})=0$.

In light of Theorem 2, we are entitled to define the set $\mathfrak{M}(G)$ as follows:

$$
\mathfrak{M}(G)=\left\{m \in M(G), \exists A, \forall m^{\prime} \in M(G),\|m\|(A)=0 \text { and }\left\|m^{\prime}(A)\right\|=0\right\} .
$$

Equivalently

$$
\mathfrak{M}(G)=\left\{m \in M(G) \mid \forall m^{\prime} \in M(G), m \perp m^{\prime}\right\} .
$$

From here we can see that $\perp$ is the characteristic condition for foreign vector measures.

Theorem 3. Any linear combination of vector measures is a vector measure.

Proof. To make it simple, let's take two vector measures $m_{1}$ and $m_{2}$ and show that $\alpha m_{1}+\beta m_{2} \in M(G)$ with $\alpha$ and $\beta$ two scalars. From Definition 2, we have:

$$
\begin{aligned}
& m_{1} \in M(G) \Longrightarrow \forall K_{1} \text { compact } \subset G \exists a_{K_{1}}>0,\left\|m_{1}(f)\right\|_{F} \leq a_{K_{1}}\|f\|_{\infty} . \\
& m_{2} \in M(G) \Longrightarrow \forall K_{2} \text { compact } \subset G \exists a_{K_{2}}>0,\left\|m_{2}(f)\right\|_{F} \leq a_{K_{2}}\|f\|_{\infty} .
\end{aligned}
$$

Now

$$
\begin{aligned}
\left\|\left(\alpha m_{1}+\beta m_{2}\right)(f)\right\|_{F} & =\left\|\alpha m_{1}(f)+\beta m_{2}(f)\right\|_{F} \\
& \leq\left\|\alpha m_{1}(f)\right\|_{F}+\left\|\beta m_{2}(f)\right\|_{F} \\
& \leq|\alpha|\left\|m_{1}(f)\right\|_{F}+|\beta|\left\|m_{2}(f)\right\|_{F} \\
& \leq|\alpha| a_{K_{1}}\|f\|_{\infty}+|\beta| a_{K_{2}}\|f\|_{\infty} \\
& \leq\left(|\alpha| a_{K_{1}}+|\beta| a_{K_{2}}\right)\|f\|_{\infty} .
\end{aligned}
$$

By setting $A_{K}=|\alpha| a_{K_{1}}+|\beta| a_{K_{2}}$ and $K=K_{1} \cap K_{2}$, we have

$$
\left\|\left(\alpha m_{1}+\beta m_{2}\right)(f)\right\| \leq A_{K}\|f\|_{\infty}, \forall f \in K .
$$

Hence the result.

\section{Main Result}

In this section, we prove all the operations with the operator $\perp$ characterizing the foreign vector measures. We take $\mathfrak{M}(G)$ provided with + and $\perp$.

Theorem 4. Let $m_{1}, m_{2}, m_{3} \in \mathfrak{M}(G)$, then

1. $m_{1} \perp m_{1} \Rightarrow m_{1}=0$.

2. $m_{1} \perp m_{2} \Leftrightarrow m_{2} \perp m_{1}$.

3. $m_{1} \perp m_{2} \Leftrightarrow\left\|m_{1}\right\| \perp\left\|m_{2}\right\|$.

4. $m_{1} \perp m_{3}$ and $m_{2} \perp m_{3} \Rightarrow\left(\alpha m_{1}+\beta m_{3}\right) \perp m_{3}$ for all $\alpha$ and $\beta$ scalars.

Proof. 1. If $m_{1} \perp m_{1}$, then there exist $A \subset G$ such that $\left\|m_{1}\right\|(A)=0$ and $\left\|m_{1}\right\|(\bar{A})=0$. Suppose $m_{1} \neq 0$. Since

$$
m_{1}(\bar{A})=m_{1}(G \backslash A)=m_{1}(G)-m_{1}(A)
$$

implies

$$
0=m_{1}(G)-m_{1}(A)
$$

implies

$$
m_{1}(G)=m_{1}(A) \neq 0
$$


and

$$
\left\|m_{1}\right\|(A) \neq 0
$$

which is absurd. Hence $m_{1}=0$.

2. Suppose that $m_{1} \perp m_{2}$

$$
\begin{gathered}
m_{1} \perp m_{2} \Leftrightarrow \exists A \subset G\|\| m_{1} \|(A)=0 \text { and }\left\|m_{2}\right\|(\bar{A})=0 \\
\Leftrightarrow\left\|m_{2}\right\|(\bar{A})=0 \text { and }\left\|m_{1}\right\|(A)=0 \\
\Leftrightarrow\left\|m_{2}\right\|(B)=0 \text { and }\left\|m_{1}\right\|(\bar{B})=0 \\
\Leftrightarrow m_{2} \perp m_{1}
\end{gathered}
$$

3. Suppose that $m_{1} \perp m_{2}$

$$
\begin{aligned}
& m_{1} \perp m_{2} \Leftrightarrow \exists A \subset G \mid\left\|m_{1}\right\|(A)=0 \text { and }\left\|m_{2}\right\|(\bar{A})=0 \\
\Leftrightarrow \quad & \left\|m_{1}\right\| \perp\left\|m_{2}\right\| \text { according to (4) Proposition } 1 .
\end{aligned}
$$

4. Suppose that $m_{1} \perp m_{3}$ and $m_{2} \perp m_{3} ; \quad \alpha$ and $\beta$ scalars

$$
\begin{aligned}
& m_{1} \perp m_{3} \Leftrightarrow \exists A \subset G \mid\left\|m_{1}\right\|(A)=0 \text { and }\left\|m_{3}\right\|(\bar{A})=0 \\
& m_{2} \perp m_{3} \Leftrightarrow \exists B \subset G \mid\left\|m_{2}\right\|(B)=0 \text { and }\left\|m_{3}\right\|(\bar{B})=0 \\
& m_{3}(\overline{A \cap B})=m_{3}(\bar{A} \cup \bar{B})=m_{3}(\bar{A})+m_{3}(\bar{B})=0 \quad \text { according to the hypothesis. }
\end{aligned}
$$

Let be $\alpha \neq 0$ and $\beta \neq 0$

$$
\left\|\alpha m_{1}\right\|(A)=0 \text { and }\left\|\beta m_{2}\right\|(B)=0 .
$$

Let be $\Omega \subset A \cap B$ then $m_{1}(\Omega)=0$ and $m_{2}(\Omega)=0$

$$
\left\|\alpha m_{1}+\beta m_{2}\right\|(A \cap B)=\sup _{\Omega \subset A \cap B}\left\{\left\|\left(\alpha m_{1}+\beta m_{2}\right)(\Omega)\right\|\right\}=0 .
$$

We have: $\left\|\alpha m_{1}+\beta m_{2}\right\|(A \cap B)=0$ and $\left\|m_{3}\right\|(\overline{A \cap B})=0$.

\section{Theorem 5.}

If $m_{1}, m_{2}, m_{3} \in \mathfrak{M}(G)$ and $m_{1} \perp m_{3}, m_{2} \perp m_{3}$ then $\left(\alpha m_{1}+\beta m_{3}\right) \perp m_{3} \forall \alpha$ and $\beta$ nonzero scalars.

Proof. Suppose that $m_{1}, m_{2}, m_{3} \in \mathfrak{M}(G)$ and $m_{1} \perp m_{3}, m_{2} \perp m_{3}$. Also since $\alpha$ and $\beta$ nonzero scalars, so $\alpha m_{1}+\beta m_{2} \in M(G)$ according to the Theorem 3 .

Now, we show that $\alpha m_{1}+\beta m_{2} \in \mathfrak{M}(G)$, by proving that $\left(\alpha m_{1}+\beta m_{3}\right) \perp m_{3}$.

According to the hypothesis

$$
m_{1} \perp m_{3} \Leftrightarrow \exists A \subset G \mid\left\|m_{1}\right\|(A)=0 \text { and }\left\|m_{3}\right\|(\bar{A})=0,
$$

then

$$
m_{2} \perp m_{3} \Leftrightarrow \exists B \subset G \mid\left\|m_{2}\right\|(B)=0 \text { and }\left\|m_{3}\right\|(\bar{B})=0 .
$$

We get easily:

$$
\begin{aligned}
m_{3}(\overline{A \cap B}) & =m_{3}(\bar{A} \cup \bar{B})(1) \\
& =m_{3}(\bar{A})+m_{3}(\bar{B}) \\
& =0 \text { according to the hypothesis. } \\
\Rightarrow\left\|m_{3}\right\|(\overline{A \cap B}) & =0
\end{aligned}
$$

Let $\alpha \neq 0$ and $\beta \neq 0$ with $\left\|\alpha m_{1}\right\|(A)=0$ and $\left\|\beta m_{2}\right\|(B)=0$ 
Let $\Omega \subset A \cap B$, then if

$$
\begin{aligned}
& \Omega \subset A \cap B \Rightarrow \Omega \subset A \text { then } m_{1}(\Omega)=0, \\
& \Omega \subset A \cap B \Rightarrow \Omega \subset B \text { then } m_{2}(\Omega)=0 \text {. } \\
& \left\|\alpha m_{1}+\beta m_{2}\right\|(A \cap B)=\sup \left\{\left\|\left(\alpha m_{1}+\beta m_{2}\right)(\Omega)\right\| ; \Omega \subset A \cap B\right\} \\
& =\sup \left\{\left\|\alpha m_{1}(\Omega)+\beta m_{2}(\Omega)\right\| ; \Omega \subset A \cap B\right\} \text { (2) } \\
& =0
\end{aligned}
$$

By choosing $A \cap B$, we have

$$
\left.\left\|\alpha m_{1}+\beta m_{2}\right\|(A \cap B)=0 \text { and }\left\|m_{3}\right\|(\overline{A \cap B})=0 \text { (according to }(10)\right) .
$$

Then $\left(\alpha m_{1}+\beta m_{3}\right) \perp m_{3}$.

Even if the characteristic relation $\perp$ is symmetrical or permutable by Theorem 4(2), we find it necessary to inform you that, it is neither associative nor transitive. Its demonstration is tedious we prefer to offer it in our next article.

Author Contributions: All authors contributed equally to the writing of this paper. All authors read and approved the final manuscript.

Conflicts of Interest: "The authors declare no conflict of interest."

\section{References}

[1] Monfort, J. A. (2019). Mesures Etrangeres, (A5). Monfort Dicostat2005, 2005-2019.

[2] Diestel, J., \& Uhl, J. J. (1977). Vector Measures. American Mathematical Society.

[3] Rudin, W (1980). Real and complex analysis. Masson Publishing USA inc.

[4] Radon, J. (1913). Theory and applications of the absolutely additive set function. Série Math-Naturwiss, 122, 1295-1438.

[5] Douhadji, A., \& Awussi, Y. (2020). Decomposition of vector measure. Theoretical Mathematics and Applications, 10(2), 9-13.

[6] Awussi, Y. (2013). On the absolute continuity of vector measure. Theoretical Mathematics and Applications, 3(4), 41-45.

(C) 2020 by the authors; licensee PSRP, Lahore, Pakistan. This article is an open access article distributed under the terms and conditions of the Creative Commons Attribution (CC-BY) license (http://creativecommons.org/licenses/by/4.0/). 